\title{
Some Properties of Analytic Functions with the Fixed Second Coefficients
}

\author{
Oh Sang Kwon \\ Department of Mathematics, Kyungsung University, Busan, Korea \\ Email: oskwon@ks.ac.kr
}

Received 20 March 2014; revised 20 April 2014; accepted 26 April 2014

Copyright (C) 2014 by author and Scientific Research Publishing Inc.

This work is licensed under the Creative Commons Attribution International License (CC BY).

http://creativecommons.org/licenses/by/4.0/

c) (i) Open Access

\begin{abstract}
In this paper, we investigate some argument properties for analytic functions with fixed second coefficient and positive real part. And we apply the argument properties to the functions that are analytic and normalized. In particular, the order of strongly starlikeness of strongly convex functions with fixed second coefficients is given.
\end{abstract}

\section{Keywords}

Analytic Functions, Argument Estimate, Fixed Second Coefficients, Strongly Starlike Functions, Strongly Convex Functions

\section{Introduction}

Let $\mathfrak{A}$ be the set of functions $f$ that are analytic in $\mathbb{U}$ and normalized by

$$
f(z)=z+\sum_{n=2}^{\infty} a_{n} z^{n}
$$

And let $\mathfrak{P}$ be the class of all functions $p$ that are analytic and have positive real part in $\mathbb{U}$ with $p(0)=1$.

Nunokawa investigated some properties of analytic functions which are not Caratheodory, that is, which are not in $\mathfrak{P}$. Furthermore, he has found the order of strongly starlikeness of strongly convex functions in $\mathfrak{A}$ (see: [1] [2]).

Now, for a fixed $\beta \in \mathbb{C}$, let $\mathfrak{P}(\beta)$ consist of functions $p \in \mathfrak{P}$ of the form

$$
p(z)=1+\beta z+p_{2} z^{2}+\cdots .
$$

And let $\mathfrak{A}(\beta)$ consist of analytic functions $f$ of the form 


$$
f(z)=z+\beta z^{2}+a_{3} z^{3}+\cdots,
$$

where the second coefficient $\beta \in \mathbb{C}$ is fixed constant.

In [3] [4], Ali et al. have extended the theory of differential subordination developed by Miller and Mocanu [5], to the functions with fixed second coefficients. And Lee et al. [6] and Nagpal et al. [7] have applied the results, to obtain several extensions of properties for univalent functions with fixed second coefficients.

In this paper, we investigate some argument properties for analytic functions $\beta \in \mathbb{C}$ with fixed second coefficients and positive real part. And we apply our results to the normalized univalent functions with fixed second coefficients.

We need the following Lemma for functions with fixed initial coefficient.

Lemma 1 [3] Let $z_{0}=r_{0} \mathrm{e}^{i \theta_{0}} \quad\left(r_{0}<1\right)$ and $g(z)=g_{n} z^{n}+g_{n+1} z^{n+1}+\cdots$ be continuous in $\overline{\mathbb{U}_{r_{0}}}$, analytic in $\mathbb{U}_{r_{0}} \cup\left\{z_{0}\right\}$ with $g(z) \neq 0$ and $n \geq 1$. If

$$
\left|g\left(z_{0}\right)\right|=\max _{|z| \leq r_{0}}|g(z)|,
$$

then

$$
\frac{z_{0} g^{\prime}\left(z_{0}\right)}{g^{\prime}\left(z_{0}\right)}=m \text { and } \operatorname{Re}\left\{1+\frac{z_{0} g^{\prime \prime}\left(z_{0}\right)}{g^{\prime}\left(z_{0}\right)}\right\} \geq m \text {, }
$$

where

$$
m \geq n+\frac{\left|g\left(z_{0}\right)\right|-\left|g_{n}\right| r_{0}^{n}}{\left|g\left(z_{0}\right)\right|+\left|g_{n}\right| r_{0}^{n}} .
$$

Here, we note that the inequality (1) implies that

$$
m \geq n+\frac{\left|g\left(z_{0}\right)\right|-\left|g_{n}\right|}{\left|g\left(z_{0}\right)\right|+\left|g_{n}\right|},
$$

since $r_{0}<1$.

\section{Lemmas}

Lemma 2 Let $p(z)=1+\beta z+p_{2} z^{2}+\cdots$ be analytic in $\mathbb{U}$ and $p(z) \neq 0$ in $\mathbb{U}$. Suppose that there exists a point $z_{0} \in \mathbb{U}$ such that

$$
\operatorname{Re}\{p(z)\}>0 \text { for }|z|<\left|z_{0}\right|
$$

and

$$
\operatorname{Re}\left\{p\left(z_{0}\right)\right\}=0 \text { and } p\left(z_{0}\right) \neq 0
$$

Then

$$
\frac{z_{0} p^{\prime}\left(z_{0}\right)}{p\left(z_{0}\right)}=i k
$$

where $k \in \mathbb{R}$ and $|k| \geq 4 /(2+|\beta|)$.

Proof. Let us put

$$
\phi(z)=\frac{1-p(z)}{1+p(z)} \quad(z \in \mathbb{U}) .
$$

Then $\phi(0)=0,|\phi(z)|<1$ for $|z|<\left|z_{0}\right|$ and $\left|\phi\left(z_{0}\right)\right|=1$. And we note that

$$
\phi(z)=-\frac{1}{2} \beta z+\left(\frac{1}{4} \beta^{2}-\frac{1}{2} p_{2}\right) z^{2}+\cdots .
$$


By Lemma 1, we have

$$
\frac{z_{0} \phi^{\prime}\left(z_{0}\right)}{\phi\left(z_{0}\right)} \geq \frac{4}{2+|\beta|}
$$

Hence

$$
\frac{z_{0} \phi^{\prime}\left(z_{0}\right)}{\phi\left(z_{0}\right)}=\frac{-2 z_{0} p^{\prime}\left(z_{0}\right)}{1+\left|p\left(z_{0}\right)\right|^{2}} \geq \frac{4}{2+|\beta|}
$$

And this inequality implies $z_{0} p^{\prime}\left(z_{0}\right)$ is a negative real number which satisfies

$$
z_{0} p^{\prime}\left(z_{0}\right) \leq \frac{-2}{2+|\beta|}\left(1+\left|P\left(z_{0}\right)\right|^{2}\right) .
$$

Now, we put $p\left(z_{0}\right)=i a$. For the case $a>0$,

$$
\operatorname{Im}\left\{\frac{z_{0} p^{\prime}\left(z_{0}\right)}{p\left(z_{0}\right)}\right\}=-\frac{z_{0} p^{\prime}\left(z_{0}\right)}{\left|p\left(z_{0}\right)\right|} \geq \frac{2}{2+|\beta|} \frac{1+\left|p\left(z_{0}\right)\right|^{2}}{\left|p\left(z_{0}\right)\right|}=\frac{2}{2+|\beta|}\left(a+\frac{1}{a}\right) .
$$

For the case $a<0$,

$$
\operatorname{Im}\left\{\frac{z_{0} p^{\prime}\left(z_{0}\right)}{p\left(z_{0}\right)}\right\}=-\frac{z_{0} p^{\prime}\left(z_{0}\right)}{\left|p\left(z_{0}\right)\right|} \leq-\frac{2}{2+|\beta|} \frac{1+\left|p\left(z_{0}\right)\right|^{2}}{\left|p\left(z_{0}\right)\right|}=\frac{2}{2+|\beta|}\left(a+\frac{1}{a}\right) .
$$

Hence, by (2) and (3),

$$
\frac{z_{0} p^{\prime}\left(z_{0}\right)}{p\left(z_{0}\right)}=i k
$$

where $k \in \mathbb{R}$ and

$$
|k| \geq \frac{2}{2+|\beta|}\left(a+\frac{1}{a}\right),
$$

Hence the proof of Lemma 2 is completed.

Lemma 3 Let $p(z)=1+\beta z+p_{2} z^{2}+\cdots$ be analytic in $\mathbb{U}$ and $p(z) \neq 0$ in $\mathbb{U}$. Suppose that there exists a $z_{0} \in \mathbb{U}$ such that

$$
|\arg \{p(z)\}|<\frac{\pi}{2} \alpha \text { for }|z|<\left|z_{0}\right|
$$

and

$$
\left|\arg \left\{p\left(z_{0}\right)\right\}\right|=\frac{\pi}{2} \alpha, \quad \alpha>0 .
$$

Then

$$
\frac{z_{0} p^{\prime}\left(z_{0}\right)}{p\left(z_{0}\right)}=i k \alpha
$$

where

$$
k \geq \frac{2}{2+|\beta|}\left(a+\frac{1}{a}\right), \text { when } \arg \left\{p\left(z_{0}\right)\right\}=\frac{\pi}{2} \alpha
$$

and

$$
k \leq-\frac{2}{2+|\beta|}\left(a+\frac{1}{a}\right) \text {, when } \arg \left\{p\left(z_{0}\right)\right\}=-\frac{\pi}{2} \alpha
$$


with

$$
p\left(z_{0}\right)^{1 / \alpha}= \pm i a .
$$

Proof. Let us put

$$
q(z)=p(z)^{1 / \alpha} .
$$

Then

$$
\operatorname{Re}\{q(z)\}>0 \text { for }|z|<\left|z_{0}\right|
$$

and

$$
\operatorname{Re}\left\{q\left(z_{0}\right)\right\}=0 .
$$

Let us put

$$
q\left(z_{0}\right)= \pm i a, \quad a>0 .
$$

Applying Lemma 2, we get

$$
\frac{z_{0} q^{\prime}\left(z_{0}\right)}{q\left(z_{0}\right)}=\frac{z_{0} p^{\prime}\left(z_{0}\right) p\left(z_{0}\right)^{(1 / \alpha)-1}}{\alpha p\left(z_{0}\right)^{1 / \alpha}}=\frac{z_{0} p^{\prime}\left(z_{0}\right)}{\alpha p\left(z_{0}\right)}=i k,
$$

where $k \in \mathbb{R}$ with

$$
k \geq \frac{2}{2+|\beta|}\left(a+\frac{1}{a}\right), \text { for } q\left(z_{0}\right)=i a
$$

and

$$
k \leq-\frac{2}{2+|\beta|}\left(a+\frac{1}{a}\right), \text { for } q\left(z_{0}\right)=-i a
$$

\section{Argument Estimates for Functions with Fixed Second Coefficient}

Theorem 4 Let $0 \leq \gamma \leq 1$ and $p \in \mathfrak{P}(\beta)$ satisfy

$$
\left|\arg \left\{p(z)+(1-\gamma) \frac{z p^{\prime}(z)}{p(z)}\right\}\right|<\frac{\pi}{2} \delta \quad(z \in \mathbb{U}) .
$$

Then

$$
|\arg \{p(z)\}|<\frac{\pi}{2} \eta \quad(z \in \mathbb{U}),
$$

where

$$
\delta=\eta+\frac{2}{\pi} \arctan \left\{\frac{4 \eta(1-\gamma) \sin (\pi(1-\eta) / 2)}{(2+|\beta|)(1+\eta)^{(1+\eta) / 2}(1-\eta)^{(1-\eta) / 2}+4 \eta(1-\gamma) \cos (\pi(1-\eta) / 2)}\right\} .
$$

Proof. Suppose that there exists a point $z_{0} \in \mathbb{U}$ such that

$$
|\arg \{p(z)\}|<\frac{\pi}{2} \eta \text { for }|z|<\left|z_{0}\right|
$$

and

$$
\left|\arg \left\{p\left(z_{0}\right)\right\}\right|=\frac{\pi}{2} \eta \quad(0<\eta \leq 1) .
$$

By Lemma 3, we can obtain that 


$$
\frac{z_{0} p^{\prime}\left(z_{0}\right)}{p\left(z_{0}\right)}=i k \eta
$$

where

$$
k \geq \frac{2}{2+|\beta|}\left(a+\frac{1}{a}\right) \text {, when } \arg \left\{p\left(z_{0}\right)\right\}=\frac{\pi}{2} \eta
$$

and

$$
k \leq-\frac{2}{2+|\beta|}\left(a+\frac{1}{a}\right) \text {, when } \arg \left\{p\left(z_{0}\right)\right\}=-\frac{\pi}{2} \eta
$$

with $p\left(z_{0}\right)^{1 / \eta}= \pm i a$. For the case $p\left(z_{0}\right)=(i a)^{\eta}(a>0)$,

$$
\begin{aligned}
& \arg \left\{p\left(z_{0}\right)+(1-\gamma) \frac{z_{0} p^{\prime}\left(z_{0}\right)}{p\left(z_{0}\right)}\right\} \\
& =\arg \left\{p\left(z_{0}\right)\right\}+\arg \left\{1+(1-\gamma) \frac{z_{0} p^{\prime}\left(z_{0}\right)}{p\left(z_{0}\right)}\right\}=\frac{\pi}{2} \eta+\arg \left\{1+(1-\gamma) \frac{i \eta k}{(i a)^{\eta}}\right\} \\
& =\frac{\pi}{2} \eta+\arctan \left\{\frac{(1-\gamma) \eta k \sin (\pi(1-\eta) / 2) / a^{\eta}}{1+\left((1-\gamma) \eta k \cos (\pi(1-\eta) / 2) / a^{\eta}\right)}\right\} \\
& \geq \frac{\pi}{2} \eta+\arctan \left\{\frac{\frac{2 \eta(1-\gamma)}{2+|\beta|}\left[\left(\frac{1+\eta}{1-\eta}\right)^{(1-\eta) / 2}+\left(\frac{1-\eta}{1+\eta}\right)^{(1+\eta) / 2}\right] \sin (\pi(1-\eta) / 2)}{1+\frac{2 \eta(1-\gamma)}{2+|\beta|}\left[\left(\frac{1+\eta}{1-\eta}\right)^{(1-\eta) / 2}+\left(\frac{1-\eta}{1+\eta}\right)^{(1+\eta) / 2}\right] \cos (\pi(1-\eta) / 2)}\right\} \\
& =\frac{\pi}{2} \eta+\arctan \left\{\frac{4 \eta(1-\gamma) \sin (\pi(1-\eta) / 2)}{(2+|\beta|)(1+\eta)^{(1+\eta) / 2}(1-\eta)^{(1-\eta) / 2}+4 \eta(1-\gamma) \cos (\pi(1-\eta) / 2)}\right\}=\frac{\pi}{2} \delta,
\end{aligned}
$$

which is a contradiction to the assumption. For the case $p\left(z_{0}\right)=(-i a)^{\eta}(a>0)$, using the same method, we can obtain a contradiction to the assumption.

Remark 5 If $|\beta|=2$, then Theorem 4 reduces the result in [[8], Theorem 3].

Theorem 6 Let $\gamma>0$ and $p \in \mathfrak{P}(\beta)$ satisfy

$$
\left|\arg \{p(z)\}+\gamma \arg \left\{1+\frac{z p^{\prime}(z)}{p^{2}(z)}\right\}\right|<\frac{\pi}{2} \delta \quad(z \in \mathbb{U}) .
$$

Then

$$
|\arg \{p(z)\}|<\frac{\pi}{2} \eta \quad(z \in \mathbb{U})
$$

where

$$
\delta=\eta+\frac{2 \gamma}{\pi} \arctan \left\{\frac{4 \eta \sin (\pi(1-\eta) / 2)}{(2+|\beta|)(1+\eta)^{(1+\eta) / 2}(1-\eta)^{(1-\eta) / 2}+4 \eta \cos (\pi(1-\eta) / 2)}\right\} .
$$

Proof. If there exists a point $z_{0} \in \mathbb{U}$ such that

$$
|\arg \{p(z)\}|<\frac{\pi}{2} \eta \quad \text { for }|z|<\left|z_{0}\right|
$$

and 


$$
\left|\arg \left\{p\left(z_{0}\right)\right\}\right|=\frac{\pi}{2} \eta \quad(0<\eta \leq 1),
$$

then Lemma 3 gives us that

$$
\frac{z_{0} p^{\prime}\left(z_{0}\right)}{p\left(z_{0}\right)}=i k \eta
$$

If $\arg \left\{p\left(z_{0}\right)\right\}=\pi \eta / 2$, then we have $p\left(z_{0}\right)^{1 / \eta}=i a(a>0)$. Therefore, we see that

$$
p\left(z_{0}\right)\left(1+\frac{z_{0} p^{\prime}\left(z_{0}\right)}{p^{2}\left(z_{0}\right)}\right)^{\gamma}=a^{\eta} \mathrm{e}^{i \eta \pi / 2}\left(1+\frac{k \eta}{a^{\eta}} \mathrm{e}^{i(1-\eta) \pi / 2}\right)^{\gamma}
$$

with

$$
k \geq \frac{2}{2+|\beta|}\left(a+\frac{1}{a}\right)
$$

Hence

$$
\frac{k \eta}{a^{\eta}} \geq \frac{2 \eta}{2+|\beta|}\left(a^{1-\eta}+a^{-1-\eta}\right) .
$$

Now, we define a function $g:(0, \infty) \rightarrow \mathbb{R}$ by

$$
g(a)=a^{1-\eta}+a^{-1-\eta} .
$$

Then

$$
g^{\prime}(a)=\frac{1-\eta}{2 a^{\eta+2}}\left(a^{2}-\frac{1+\eta}{1-\eta}\right) .
$$

Hence $g(a)$ takes the minimum value at $a=((1+\eta)(1-\eta))^{1 / 2}$. Therefore,

$$
\begin{aligned}
\frac{k \eta}{a^{\eta}} & \geq \frac{2 \eta}{2+|\beta|} g(a) \geq \frac{2 \eta}{2+|\beta|} g\left(\left(\frac{1+\eta}{1-\eta}\right)^{\frac{1}{2}}\right) \\
& =\frac{2 \eta}{2+|\beta|}\left\{\left(\frac{1+\eta}{1-\eta}\right)^{\frac{1}{2}(1-\eta)}+\left(\frac{1-\eta}{1+\eta}\right)^{\frac{1}{2}(1+\eta)}\right\} .
\end{aligned}
$$

Thus we have

$$
\begin{aligned}
& \arg \left\{p\left(z_{0}\right)\right\}+\gamma \arg \left\{1+\frac{z_{0} p^{\prime}\left(z_{0}\right)}{p\left(z_{0}\right)}\right\}=\frac{\pi}{2} \eta+\gamma \arg \left\{1+\frac{k \eta}{a^{\eta}} \mathrm{e}^{i(1-\eta) \pi / 2}\right\} \\
& \geq \frac{\pi}{2} \eta+\gamma \arctan \left\{\frac{4 \eta \sin (\pi(1-\eta) / 2)}{(2+|\beta|)(1+\eta)^{(1+\eta) / 2}(1-\eta)^{(1-\eta) / 2}+4 \eta \cos (\pi(1-\eta) / 2)}\right\}=\frac{\pi}{2} \delta,
\end{aligned}
$$

which contradicts the condition (5). And if $\arg \left\{p\left(z_{0}\right)\right\}=-\pi \eta / 2$, applying the same method we have

$$
\arg \left\{p\left(z_{0}\right)\right\}+\gamma \arg \left\{1+\frac{z_{0} p^{\prime}\left(z_{0}\right)}{p\left(z_{0}\right)}\right\} \leq-\frac{\pi}{2} \delta,
$$

which contradicts the condition (5). And this completes the proof of the Theorem 6 .

Remark 7 If $|\beta|=2$, then Theorem 6 reduces the result in [[8], Theorem 1].

Theorem 8 Let $p \in \mathfrak{P}(\beta)$ and 


$$
\left|\arg \left\{z p^{\prime}(z)+p^{2}(z)+\alpha p(z)\right\}\right|<\delta(\rho):=\frac{\pi}{2} \rho+\arctan \left(\frac{4 \rho}{\alpha(2+|\beta|)}\right) \quad(z \in \mathbb{U}),
$$

for some $\alpha>0, \quad \rho \quad\left(0<\rho \leq \rho_{0}\right)$, where $\rho_{0} \quad\left(0<\rho_{0}<1\right)$ is given by

$$
\tan \left(\frac{\pi}{2} \rho_{0}\right)=\frac{4 \rho_{0}}{\alpha(2+|\beta|)}
$$

Then

$$
|\arg \{p(z)\}|<\frac{\pi}{2} \rho \quad(z \in \mathbb{U}) .
$$

Proof. Suppose that there exists a $z_{0} \in \mathbb{U}$ such that

$$
|\arg \{p(z)\}|<\frac{\pi}{2} \rho \text { for }|z|<\left|z_{0}\right|
$$

and

$$
\left|\arg \left\{p\left(z_{0}\right)\right\}\right|=\frac{\pi}{2} \rho .
$$

By Lemma 3, we can obtain that

$$
\frac{z_{0} p^{\prime}\left(z_{0}\right)}{p\left(z_{0}\right)}=i k \rho
$$

where $k \in \mathbb{R}$ with

$$
k \geq \frac{2}{2+|\beta|}\left(a+\frac{1}{a}\right), \quad \text { when } p\left(z_{0}\right)^{1 / \rho}=i a
$$

and

$$
k \leq-\frac{2}{2+|\beta|}\left(a+\frac{1}{a}\right), \quad \text { when } p\left(z_{0}\right)^{1 / \rho}=-i a \quad(a>0) .
$$

For the case $p\left(z_{0}\right)=(i a)^{\rho}$,

$$
\begin{aligned}
\arg \left\{z_{0} p^{\prime}\left(z_{0}\right)+p^{2}\left(z_{0}\right)+\alpha p\left(z_{0}\right)\right\} & =\arg \left\{p\left(z_{0}\right)\right\}+\arg \left\{\frac{z_{0} p^{\prime}\left(z_{0}\right)}{p\left(z_{0}\right)}+p\left(z_{0}\right)+\alpha\right\} \\
& =\frac{\pi}{2} \rho+\arg \left\{i \rho k+a^{\rho} \mathrm{e}^{i \pi \rho / 2}+\alpha\right\} \\
& =\frac{\pi}{2} \rho+\arg \left\{\alpha+a^{\rho} \cos (\pi \rho / 2)+i\left(\rho k+a^{\rho} \sin (\pi \rho / 2)\right)\right\} \\
& =\frac{\pi}{2} \rho+\arctan \left\{\frac{\rho k+a^{\rho} \sin (\pi \rho / 2)}{\alpha+a^{\rho} \cos (\pi \rho / 2)}\right\} \\
& \geq \frac{\pi}{2} \rho+\arctan \left\{\frac{\frac{4 \rho}{2+|\beta|}+a^{\rho} \sin (\pi \rho / 2)}{\alpha+a^{\rho} \cos (\pi \rho / 2)}\right\},
\end{aligned}
$$

since

$$
k \geq \frac{2}{2+|\beta|}\left(a+\frac{1}{a}\right) \geq \frac{4}{2+|\beta|}
$$


Now, we define

$$
g(a)=\frac{\frac{4 \rho}{2+|\beta|}+a^{\rho} \sin (\pi \rho / 2)}{\alpha+a^{\rho} \cos (\pi \rho / 2)} .
$$

Then

$$
g^{\prime}(a)=\frac{\alpha \rho a^{\rho-1} \cos (\pi \rho / 2)\left(\tan (\pi \rho / 2)-\frac{4 \rho}{\alpha(2+|\beta|)}\right)}{\left(\alpha+a^{\rho} \cos (\pi \rho / 2)\right)^{2}} .
$$

Define

$$
h(\rho)=\tan (\pi \rho / 2)-\frac{4 \rho}{\alpha(2+|\beta|)} \quad\left(0<\rho \leq \rho_{0}<1\right) .
$$

Then $h(0)=0$ and $h\left(\rho_{0}\right)=0$. Furthermore,

$$
h^{\prime \prime}(\rho)=\frac{\pi^{2}}{2} \sec ^{2}\left(\frac{\pi}{2} \rho\right) \tan \left(\frac{\pi}{2} \rho\right),
$$

for all $0<\rho \leq \rho_{0}<1$. Hence $g^{\prime}(a) \leq 0$ for all $a>0$. And

$$
g(a) \geq \inf _{a>0} g(a)=\frac{4 \rho}{\alpha(2+|\beta|)} .
$$

By (8), we have

$$
\arg \left\{z_{0} p^{\prime}\left(z_{0}\right)+p^{2}\left(z_{0}\right)+\alpha p\left(z_{0}\right)\right\} \geq \frac{\pi}{2} \rho+\arctan \left(\frac{4 \rho}{\alpha(2+|\beta|)}\right),
$$

which is a contradiction to the hypothesis. For the case $p\left(z_{0}\right)=(-i a)^{\rho} \quad(a>0)$, using the same method, we can obtain a contradiction to the assumption.

Remark 9 If $|\beta|=2$, then Theorem 8 reduces the result in [[9], Theorem 2.1].

\section{Corollaries}

For a function $f \in \mathfrak{A}, f$ is called strongly starlike of order $\eta,(0<\eta \leq 1)$, if

$$
\left|\arg \left\{\frac{z f^{\prime}(z)}{f(z)}\right\}\right|<\frac{\pi}{2} \eta \quad(z \in \mathbb{U}) .
$$

And $f$ is called strongly convex of order $\eta,(0<\eta \leq 1)$, if

$$
\left|\arg \left\{1+\frac{z f^{\prime \prime}(z)}{f^{\prime}(z)}\right\}\right|<\frac{\pi}{2} \eta \quad(z \in \mathbb{U}) .
$$

Using these definitions and Theorem in Section 3, we can obtain the following corollaries.

Corollary 10 Let $f \in \mathfrak{A}(\beta)$ and

$$
\left|\arg \left\{\gamma \frac{z f^{\prime}(z)}{f(z)}+(1-\gamma)\left(1+\frac{z f^{\prime \prime}(z)}{f^{\prime}(z)}\right)\right\}\right|<\frac{\pi}{2} \delta \quad(z \in \mathbb{U}) .
$$

Then $f$ is strongly starlike of order $\eta$, where $\eta$ is given by (4).

Putting $\gamma=0$ in Corollary 1 , we can obtain the following Corollary.

Corollary 11 Let $f \in \mathfrak{A}(\beta)$ be a strongly convex function of order $\delta$. Then $f$ is a strongly starlike 
function of order $\eta$, where $\eta$ is given by

$$
\delta=\eta+\frac{2}{\pi} \arctan \left\{\frac{4 \eta \sin (\pi(1-\eta) / 2)}{(2+|\beta|)(1+\eta)^{(1+\eta) / 2}(1-\eta)^{(1-\eta) / 2}+4 \eta \cos (\pi(1-\eta) / 2)}\right\} .
$$

Corollary 12 Let $\gamma>0$ and $f \in \mathfrak{A}(\beta)$ satisfy

$$
\left|\arg \left\{\left(\frac{z f^{\prime}(z)}{f(z)}\right)^{1-\gamma}\left(1+\frac{z f^{\prime \prime}(z)}{f^{\prime}(z)}\right)^{\gamma}\right\}\right|<\frac{\pi}{2} \delta \quad(z \in \mathbb{U}) .
$$

Then $f$ is strongly starlike of order $\eta$, where $\eta$ is given by (6).

Corollary 13 Let $f \in \mathfrak{A}(\beta)$ and

$$
\left|\arg \left\{\frac{z f^{\prime}(z)}{f(z)}\left(\alpha+1+\frac{z f^{\prime \prime}(z)}{f^{\prime}(z)}\right)\right\}\right|<\delta(\rho) \quad(z \in \mathbb{U}),
$$

where $\delta(\rho)$ is given by (7). Then $f$ is strongly starlike of order $\rho$.

\section{References}

[1] Nunokawa, M. (1992) On Properties of Non-Caratheodory Functions. Proceedings of the Japan Academy, Series A, Mathematical Sciences, 68, 152-153.

[2] Nunokawa, M. (1993) On the Order of Strongly Starlikeness of Strongly Convex Functions. Proceedings of the Japan Academy, Series A, Mathematical Sciences, 69, 234-237.

[3] Ali, R.M., Nagpal, S. and Ravichandran, V. (2011) Second-Order Differential Subordination for Analytic Functions with Fixed Initial Coefficient. Bulletin of the Malaysian Mathematical Sciences Society, 34, 611-629.

[4] Ali, R.M., Cho, N.E., Jain, N. and Ravichandran, V. (2012) Radii of Starlikeness and Convexity of Functions Defined by Subordination with Fixed Second Coefficients, Filomat, 26, 553-561. http://dx.doi.org/10.2298/FIL1203553A

[5] Miller, S.S. and Mocanu, P.T. (2000) Differential Subordinations. Dekker, New York.

[6] Lee, S.K., Ravichandran, V. and Supramaniam, S. (2012) Applications of Differential Subordination for Functions with Fixed Coefficient to Geometric Function Theory. arXiv:1209.0896.

[7] Nagpal, S. and Ravichandran, V. (2012) Applications of Theory of Differential Subordinatioin for Functions with Fixed Initial Coefficient to Univalent Functions. Annales Polonici Mathematici, 105, 225-238. http://dx.doi.org/10.4064/ap105-3-2

[8] Nunokawa, M., Owa, S., Hayami, T., Kuroki, K. and Uyanik, N. (2011) Some Conditions for Strongly Starlikeness of Certain Analytic Functions. International Mathematical Forum, 6, 1199-1208.

[9] Nunokawa, M., Owa, S. and Saitoh, H. (2003) Argument Estimates for Certain Analytic Functions. Proceedings of the Japan Academy, Series A, Mathematical Sciences, 79, 163-166. 